\title{
Estimates on the lower bound of the first gap
}

\author{
JUN LING
}

Dedicated to Professor Yieh-Hei Wan

\begin{abstract}
We give a new lower bound for the first gap $\lambda_{2}-\lambda_{1}$ of the Dirichlet eigenvalues of the Schrödinger operator on a bounded convex domain $\Omega$ in $\mathrm{R}^{n}$ or $\mathrm{S}^{n}$, in terms of the diameter of the domain and the global convexity.
\end{abstract}

\section{Introduction}

We give a new estimate on the lower bound of the gap of the first two Dirichlet eigenvalues of the Schrödinger operator on a bounded convex domain $\Omega$ in $\mathrm{R}^{n}$ or $\mathrm{S}^{n}$, in terms of the diameter of the domain and the global convexity. Let $\lambda_{1}$ and $\lambda_{2}$ be the first two Dirichlet eigenvalues of the Schrödinger operator $-\Delta+V$ to the eigenvalue problem

$$
-\Delta u+V u=\lambda u \text { in } \Omega, \quad u=0 \text { on } \partial \Omega,
$$

where $\Delta$ is the Laplacian on $\Omega, V: \Omega \rightarrow \mathrm{R}^{1}$ a non-negative convex smooth function, $\Omega$ is bounded and convex domain in $\mathrm{R}^{n}$ or $\mathrm{S}^{n}$, where 'convex domain' means the second fundamental form of the boundary with respect to the outward normal to the boundary is positive definite.

It is an interesting and important problem to find a lower bound for the first gap $\lambda_{2}-\lambda_{1}>0$. There has been a lot of work on this problem. See $[1,4]$ for the references. For a general bounded convex domain in $\mathrm{R}^{n}$, Singer et al. [15] showed that

$$
\lambda_{2}-\lambda_{1} \geq \frac{\pi^{2}}{4 d^{2}},
$$

where $d$ is the diameter of the domain. Yu and Zhong [19] later removed the factor 1/4. Lee and Wang [9] showed the above estimate remains true for the Laplacian on a bounded convex domain in $\mathrm{S}^{n}$. The author [11] proved that global log-convexity holds if the domain in $\mathrm{R}^{n}$ or $\mathrm{S}^{n}$ is convex, and 
therefore one has the strict lower bound

$$
\lambda_{2}-\lambda_{1}>\frac{\pi^{2}}{d^{2}} .
$$

Smits [14] gave an alternative derivation of the above result.

There are two directions for the further study of the lower bound.

One direction is motivated statistical physics. van den Berg [5] conjectured that the lower bound is $3 \pi^{2} / d^{2}$. See also Yau's Problem Section in $[13,16]$ and Ashbaugh [1]. The authors in $[2,4,3,8]$ proved the conjecture in $\mathrm{R}^{2}$ for some special symmetric domains and for some special potential $V$.

The other direction of the study is motivated by geometry. See Yau's Perspectives on Geometric Analysis [18] for details. In [17], Yau gave an interesting estimate

$$
\lambda_{2}-\lambda_{1} \geq \theta \frac{\pi^{2}}{d^{2}}+2(\cos \pi \sqrt{\theta})^{2} \alpha,
$$

where $\theta$ is any constant with $0 \leq \theta \leq 1 / 4, d$ is the diameter, and $\alpha>0$ is the global log-convexity

$$
\alpha=\inf _{x \in \Omega} \inf _{\tau \in \mathrm{T}_{x} \Omega,|\tau|=1}\left[\nabla^{2}(-\ln f)\right](\tau, \tau)(x),
$$

$f$ is a positive first eigenfunction. By $[6,11], \alpha>0$. Yau [17] gave an interesting estimate on the lower bound of $\alpha$ in terms of the potential $V$.

The above (1.2) gives $\lambda_{2}-\lambda_{1} \geq \pi^{2} / 4 d^{2}$ and $\lambda_{2}-\lambda_{1} \geq 2 \alpha$ and intermediate results between the two.

Along Yau's geometric direction and using Li-Yau [10] and Yu-Zhong [19]'s approach, in this paper we improve (1.2) to the following:

$$
\lambda_{2}-\lambda_{1} \geq \frac{\pi^{2}}{d^{2}}+0.62 \alpha .
$$

If the domain and the potential have certain symmetry, then we have

$$
\lambda_{2}-\lambda_{1} \geq \frac{\pi^{2}}{d^{2}}+\alpha .
$$

We expect that the results can be contributed to the study of the bound along the direction motivated by statistical physics as well.

Theorem 1.1. If $\Delta$ is the Laplacian in $\mathrm{R}^{n}$ or $\mathrm{S}^{n}$ and if $\lambda_{1}$ and $\lambda_{2}$ are the first two Dirichlet eigenvalues of the Schrödinger operator $-\Delta+V$ with a 
non-negative convex potential $V$ on a bounded convex domain $\Omega$ in $\mathrm{R}^{n}$ or $\mathrm{S}^{n}$, then (1.4) holds.

Theorem 1.2. If in addition to the conditions in Theorem 1.1, the domain $\Omega$ has a certain symmetry that the minimum of the ratio of eigenfunctions is the opposite of the maximum, then (1.5) holds.

Remark. The above result holds when $\Omega$ is in a manifold with non-negative Ricci curvature and positive $\alpha$.

We prove our results by using the maximum principle and test functions. In the next section, we study exact solutions of some ordinary differential equations and explore the properties of the function $\xi$ constructed by the author in [12], the Zhong-Yang function $\eta$ and the ratio $\xi / \eta$, which are essential to our construction of the required test functions. The functions $\xi$ and $\eta$ and their properties also have other important applications. We then use the exact solutions to construct the test functions in Section 3 and prove our main results. The last section is for deriving some preliminary estimates and the conditions for test functions and for proving some technical lemmas needed in the proof of the Theorem 1.1.

\section{Exact solutions of some ODEs}

Lemma 2.1. Let

$$
\xi(t)=\frac{\cos ^{2} t+2 t \sin t \cos t+t^{2}-\left(\pi^{2} / 4\right)}{\cos ^{2} t} \quad \text { on }\left[-\frac{\pi}{2}, \frac{\pi}{2}\right] .
$$

Then the function $\xi$ satisfies the following

(2.2) $\frac{1}{2} \xi^{\prime \prime} \cos ^{2} t-\xi^{\prime} \cos t \sin t-\xi=2 \cos ^{2} t \quad$ in $\left(-\frac{\pi}{2}, \frac{\pi}{2}\right)$,

(2.3) $\xi^{\prime} \cos t-2 \xi \sin t=4 t \cos t \quad$ in $\left(-\frac{\pi}{2}, \frac{\pi}{2}\right)$,

(2.4) $\int_{0}^{\pi / 2} \xi(t) d t=-\frac{\pi}{2}$

$1-\frac{\pi^{2}}{4}=\xi(0) \leq \xi(t) \leq \xi\left( \pm \frac{\pi}{2}\right)=0 \quad$ on $\left[-\frac{\pi}{2}, \frac{\pi}{2}\right]$,

$\xi^{\prime}$ is increasing on $\left[-\frac{\pi}{2}, \frac{\pi}{2}\right]$ and $\xi^{\prime}\left( \pm \frac{\pi}{2}\right)= \pm \frac{2 \pi}{3}$, 


$$
\begin{aligned}
& \xi^{\prime}(t)<0 \text { on }\left(-\frac{\pi}{2}, 0\right) \text { and } \xi^{\prime}(t)>0 \text { on }\left(0, \frac{\pi}{2}\right), \\
& \xi^{\prime \prime}\left( \pm \frac{\pi}{2}\right)=2, \xi^{\prime \prime}(0)=2\left(3-\frac{\pi^{2}}{4}\right) \text { and } \xi^{\prime \prime}(t)>0 \text { on }\left[-\frac{\pi}{2}, \frac{\pi}{2}\right], \\
& \left(\frac{\xi^{\prime}(t)}{t}\right)^{\prime}>0 \text { on }\left(0, \frac{\pi}{2}\right) \text { and } 2\left(3-\frac{\pi^{2}}{4}\right) \leq \frac{\xi^{\prime}(t)}{t} \leq \frac{4}{3} \text { on }\left[-\frac{\pi}{2}, \frac{\pi}{2}\right], \\
& \xi^{\prime \prime \prime}\left(\frac{\pi}{2}\right)=\frac{8 \pi}{15}, \quad \xi^{\prime \prime \prime}(t)<0 \text { on }\left(-\frac{\pi}{2}, 0\right) \text { and } \xi^{\prime \prime \prime}(t)>0 \text { on }\left(0, \frac{\pi}{2}\right) .
\end{aligned}
$$

Proof. For convenience, let $q(t)=\xi^{\prime}(t)$, i.e.,

$$
q(t)=\xi^{\prime}(t)=\frac{2\left(2 t \cos t+t^{2} \sin t+\cos ^{2} t \sin t-\left(\pi^{2} / 4\right) \sin t\right)}{\cos ^{3} t}
$$

Equation (2.2) and the values $\xi( \pm \pi / 2)=0, \xi(0)=1-\pi^{2} / 4$ and $\xi^{\prime}( \pm \pi / 2)=$ $\pm 2 \pi / 3$ can be verified directly from $(2.1)$ and $(2.5)$. The values of $\xi^{\prime \prime}$ at 0 and $\pm \pi / 2$ can be computed via (2.2). By $(2.3),\left(\xi(t) \cos ^{2} t\right)^{\prime}=4 t \cos ^{2} t$. Therefore $\xi(t) \cos ^{2} t=\int_{\pi / 2}^{t} 4 s \cos ^{2} s d s$, and

$$
\begin{aligned}
& \int_{-\pi / 2}^{\pi / 2} \xi(t) d t=2 \int_{0}^{\pi / 2} \xi(t) d t=-8 \int_{0}^{\pi / 2}\left(\frac{1}{\cos ^{2}(t)} \int_{t}^{\pi / 2} s \cos ^{2} s d s\right) d t \\
& \quad=-8 \int_{0}^{\pi / 2}\left(\int_{0}^{s} \frac{1}{\cos ^{2}(t)} d t\right) s \cos ^{2} s d s=-8 \int_{0}^{\pi / 2} s \cos s \sin s d s=-\pi .
\end{aligned}
$$

It is easy to see that $q$ and $q^{\prime}$ satisfy the following equations

$$
\frac{1}{2} q^{\prime \prime} \cos t-2 q^{\prime} \sin t-2 q \cos t=-4 \sin t
$$

and

$$
\frac{\cos ^{2} t}{2\left(1+\cos ^{2} t\right)}\left(q^{\prime}\right)^{\prime \prime}-\frac{2 \cos t \sin t}{1+\cos ^{2} t}\left(q^{\prime}\right)^{\prime}-2\left(q^{\prime}\right)=-\frac{4}{1+\cos ^{2} t} .
$$

The last equation implies that $q^{\prime}=\xi^{\prime \prime}$ cannot achieve its non-positive local minimum at a point in $(-\pi / 2, \pi / 2)$. On the other hand, $\xi^{\prime \prime}( \pm \pi / 2)=2$, by equation (2.2), $\xi( \pm \pi / 2)=0$ and $\xi^{\prime}( \pm \pi / 2)= \pm 2 \pi / 3$. Therefore $\xi^{\prime \prime}(t)>0$ on $[-\pi / 2, \pi / 2]$ and $\xi^{\prime}$ is increasing. Since $\xi^{\prime}(0)=0$, we have $\xi^{\prime}(t)<0$ on 
$(-\pi / 2,0)$ and $\xi^{\prime}(t)>0$ on $(0, \pi / 2)$. Similarly, from the equation

$$
\begin{aligned}
& \frac{\cos ^{2} t}{2\left(1+\cos ^{2} t\right)}\left(q^{\prime \prime}\right)^{\prime \prime}-\frac{\cos t \sin t\left(3+2 \cos ^{2} t\right)}{\left(1+\cos ^{2} t\right)^{2}}\left(q^{\prime \prime}\right)^{\prime}-\frac{2\left(5 \cos ^{2} t+\cos ^{4} t\right)}{\left(1+\cos ^{2} t\right)^{2}}\left(q^{\prime \prime}\right) \\
& \quad=-\frac{8 \cos t \sin t}{\left(1+\cos ^{2} t\right)^{2}}
\end{aligned}
$$

we obtain the results in the last line of the lemma.

Set $h(t)=\xi^{\prime \prime}(t) t-\xi^{\prime}(t)$. Then $h(0)=0$ and $h^{\prime}(t)=\xi^{\prime \prime \prime}(t) t>0$ in $(0, \pi / 2)$. Therefore $\left(\xi^{\prime}(t) / t\right)^{\prime}=h(t) / t^{2}>0$ in $(0, \pi / 2)$. Note that

$$
\frac{\xi^{\prime}(-t)}{-t}=\frac{\xi^{\prime}(t)}{t},\left.\frac{\xi^{\prime}(t)}{t}\right|_{t=0}=\xi^{\prime \prime}(0)=2\left(3-\frac{\pi^{2}}{4}\right) \quad \text { and }\left.\quad \frac{\xi^{\prime}(t)}{t}\right|_{t=\pi / 2}=\frac{4}{3} \text {. }
$$

This completes the proof of the lemma.

Lemma 2.2. Let

$$
\eta(t)=\frac{(4 / \pi) t+(4 / \pi) \cos t \sin t-2 \sin t}{\cos ^{2} t} \text { on } \quad\left[-\frac{\pi}{2}, \frac{\pi}{2}\right] .
$$

Then the function $\eta$ satisfies the following

$$
\begin{aligned}
& \frac{1}{2} \eta^{\prime \prime} \cos ^{2} t-\eta^{\prime} \cos t \sin t-\eta=-\sin t \quad \text { in }\left(-\frac{\pi}{2}, \frac{\pi}{2}\right) \\
& \eta^{\prime} \cos t-2 \eta \sin t=\frac{8}{\pi} \cos t-2 \quad \text { in }\left(-\frac{\pi}{2}, \frac{\pi}{2}\right) \\
& -1=\eta\left(-\frac{\pi}{2}\right) \leq \eta(t) \leq \eta\left(\frac{\pi}{2}\right)=1 \quad \text { on }\left[-\frac{\pi}{2}, \frac{\pi}{2}\right] \\
& 0<2\left(\frac{4}{\pi}-1\right)=\eta^{\prime}(0) \leq \eta^{\prime}(t) \leq \eta^{\prime}\left( \pm \frac{\pi}{2}\right)=\frac{8}{3 \pi} \quad \text { on }\left[-\frac{\pi}{2}, \frac{\pi}{2}\right], \\
& -\frac{1}{2}=\eta^{\prime \prime}\left(-\frac{\pi}{2}\right) \leq \eta^{\prime \prime}(t) \leq \eta^{\prime \prime}\left(\frac{\pi}{2}\right)=\frac{1}{2} \quad \text { on }\left[-\frac{\pi}{2}, \frac{\pi}{2}\right] \\
& \eta^{\prime \prime \prime}(t)>0 \text { on }\left[-\frac{\pi}{2}, \frac{\pi}{2}\right] \quad \text { and } \quad \eta^{\prime \prime \prime}\left( \pm \frac{\pi}{2}\right)=\frac{32}{15 \pi} .
\end{aligned}
$$

Proof. $\quad$ Let $p(t)=\eta^{\prime}(t)$, i.e.,

$$
p(t)=\eta^{\prime}(t)=\frac{2\left((4 / \pi) \cos t+(4 / \pi) t \sin t-\sin ^{2} t-1\right)}{\cos ^{3} t} .
$$

Equation $(2.10), \eta( \pm \pi / 2)= \pm 1, \eta^{\prime}(0)=2((4 / \pi)-1)$ and $\eta^{\prime}( \pm \pi / 2)=$ $8 / 3 \pi$ can be verified directly. We obtain $\eta^{\prime \prime}( \pm \pi / 2)= \pm 1 / 2$ from the above 
values and equation (2.10). By (2.10), $p=\eta^{\prime}, p^{\prime}=\eta^{\prime \prime}$ and $p^{\prime \prime}=\eta^{\prime \prime \prime}$ satisfy the following equations in $(-\pi / 2, \pi / 2)$

$$
\begin{aligned}
\frac{1}{2} p^{\prime \prime} \cos t-2 p^{\prime} \sin t-2 p \cos t & =-1, \\
\frac{\cos ^{2} t}{2\left(1+\cos ^{2} t\right)} p^{\prime \prime \prime}-\frac{2 \cos t \sin t}{1+\cos ^{2} t} p^{\prime \prime}-2 p^{\prime} & =-\frac{\sin t}{1+\cos ^{2} t},
\end{aligned}
$$

and

$$
\begin{aligned}
& \frac{\cos ^{2} t}{2\left(1+\cos ^{2} t\right)}\left(p^{\prime \prime}\right)^{\prime \prime}-\frac{\cos t \sin t\left(3+2 \cos ^{2} t\right)}{\left(1+\cos ^{2} t\right)^{2}}\left(p^{\prime \prime}\right)^{\prime}-\frac{2\left(5 \cos ^{2} t+\cos ^{4} t\right)}{\left(1+\cos ^{2} t\right)^{2}}\left(p^{\prime \prime}\right) \\
& \quad=-\frac{\cos t(2+\sin t)}{\left(1+\cos ^{2} t\right)^{2}}
\end{aligned}
$$

The coefficient of $\left(p^{\prime \prime}\right)$ in (2.14) is obviously negative in $(-\pi / 2, \pi / 2)$, and the right-hand side of (2.14) is also negative. So $p^{\prime \prime}$ cannot achieve its nonpositive local minimum at a point in $(-\pi / 2, \pi / 2)$. On the other hand, $p^{\prime \prime}(\pi / 2)=32 / 15 \pi>0$ (see the proof below), $p^{\prime \prime}(t)>0$ on $[-\pi / 2, \pi / 2]$. Therefore $p^{\prime}$ is increasing and $-1 / 2=p^{\prime}(-\pi / 2) \leq p^{\prime}(t) \leq p^{\prime}(\pi / 2)=1 / 2$. Note that $p^{\prime}(0)=0\left(p^{\prime}\right.$ is an odd function). So $p^{\prime}(t)>0$ on $(0, \pi / 2)$ and $p$ is increasing on $[0, \pi / 2]$. Therefore, $2(4 / \pi-1)=p(0) \leq p(t)=\eta^{\prime}(t) \leq$ $p(\pi / 2)=8 / 3 \pi$ on $[0, \pi / 2]$, and on $[-\pi / 2, \pi / 2]$ since $p$ is an even function. We now show that $p(\pi / 2)=8 / 3 \pi, p^{\prime}(\pi / 2)=1 / 2$ and $p^{\prime \prime}(\pi / 2)=32 / 15 \pi$. The first is from a direct computation by using (2.12). By (2.10),

$$
\begin{aligned}
\frac{1}{2} p^{\prime}\left(\frac{\pi}{2}\right)=\frac{1}{2} \eta^{\prime \prime}\left(\frac{\pi}{2}\right) & =\lim _{t \rightarrow \frac{\pi}{2}-} \frac{\eta^{\prime}(t) \cos t \sin t+\eta(t)-\sin t}{\cos ^{2} t} \\
& =-\frac{1}{2}\left[\eta^{\prime \prime}\left(\frac{\pi}{2}\right)-1\right] .
\end{aligned}
$$

So $p^{\prime}(\pi / 2)=1 / 2$. Similarly, by $(2.13)$,

$$
\frac{1}{2} p^{\prime \prime}\left(\frac{\pi}{2}\right)=\lim _{t \rightarrow \frac{\pi}{2}-} \frac{2 p^{\prime}(t) \sin t-1}{\cos t}+2 p\left(\frac{\pi}{2}\right)=-2 p^{\prime \prime}\left(\frac{\pi}{2}\right)+\frac{16}{3 \pi}
$$

Thus $p^{\prime \prime}(\pi / 2)=32 / 15 \pi$.

Lemma 2.3. The function $r(t)=\xi^{\prime}(t) / \eta^{\prime}(t)$ is an increasing function on $[-\pi / 2, \pi / 2]$, i.e., $r^{\prime}(t)>0$, and $|r(t)| \leq \pi^{2} / 4$ holds on $[-\pi / 2, \pi / 2]$. 
Proof. $\quad$ Let $p(t)=\eta^{\prime}(t)$ as in (2.12) and $q(t)=\xi^{\prime}(t)$. Then $r(t)=q(t) / p(t)$. It is easy to verify that $r( \pm \pi / 2)= \pm \pi^{2} / 4$. By (2.13) and (2.6),

$$
\left(\frac{1}{2}\right) p(t) r^{\prime \prime} \cos t+\left(p^{\prime}(t) \cos t-2 p(t) \sin t\right) r^{\prime}-r=-4 \sin t .
$$

Differentiating the last equation, we obtain

$$
\begin{aligned}
& {\left[\frac{1}{2} p(t) \cos t\right]\left(r^{\prime}\right)^{\prime \prime}+\left[\frac{3}{2} p^{\prime}(t) \cos t-\frac{5}{2} p(t) \sin t\right]\left(r^{\prime}\right)^{\prime}} \\
& \quad+\left[p^{\prime \prime}(t) \cos t-3 p^{\prime}(t) \sin t-2 p(t) \cos t-1\right]\left(r^{\prime}\right)=-4 \cos t .
\end{aligned}
$$

Using (2.13), the above equation becomes

$$
\begin{aligned}
& {\left[\frac{1}{2} p(t) \cos t\right]\left(r^{\prime}\right)^{\prime \prime}+\left[\frac{3}{2} p^{\prime}(t) \cos t-\frac{5}{2} p(t) \sin t\right]\left(r^{\prime}\right)^{\prime}} \\
& \quad+\left[p^{\prime}(t) \sin t+2 p(t) \cos t-3\right]\left(r^{\prime}\right)=-4 \cos t
\end{aligned}
$$

The coefficient of $\left(r^{\prime}\right)$ in (2.14) is negative, for $p^{\prime}(t) \sin t+2 p \cos t-3<$ $1 / 2+16 / 3 \pi-3<0$. This fact and the negativity of the right-hand side of (2.14) in $(-\pi / 2, \pi / 2)$ imply that $r^{\prime}$ cannot achieve its non-positive minimum on $[-\pi / 2, \pi / 2]$ at a point in $(-\pi / 2, \pi / 2)$. Now

$$
\begin{aligned}
\lim _{t \rightarrow \frac{\pi}{2}^{-}} r^{\prime}(t) & =\lim _{t \rightarrow \frac{\pi}{2}^{-}} \frac{s(t) \cos ^{2} t}{\left((4 / \pi) \cos t+(4 / \pi) t \sin t-\sin ^{2} t-1\right)^{2}} \\
& =\lim _{t \rightarrow \frac{\pi}{2}-} \frac{\left[s(t) / \cos ^{4} t\right]}{\left[\left((4 / \pi) \cos t+(4 / \pi) t \sin t-\sin ^{2} t-1\right) / \cos ^{3} t\right]^{2}} \\
& =\lim _{t \rightarrow \frac{\pi}{2}-} \frac{\left[s(t) / \cos ^{4} t\right]}{\left[(1 / 2) \eta^{\prime}(t)\right]^{2}} \\
& =\frac{(4 / 3 \pi-\pi / 12)}{(4 / 3 \pi)^{2}} \\
& >0
\end{aligned}
$$

where

$$
\begin{aligned}
s(t)= & -\frac{4}{\pi} t^{2}-t^{2} \cos t+\frac{12}{\pi} \cos ^{2} t+\frac{8}{\pi} t \sin t \cos t \\
& -\cos t \sin ^{2} t+\left(\frac{\pi^{2}}{4}-3\right) \cos t-\pi+4 t \sin t .
\end{aligned}
$$

Therefore, $r^{\prime}(t)>0$ and $r$ is an increasing function on $[-\pi / 2, \pi / 2]$. 


\section{Proof of the main result}

Let $f_{2}$ be the second eigenfunction and $f$ a positive first eigenfunction of problem (1.1). It is known that $f_{2} / f$ changes its signs in $\Omega$ (see [7]) and is smooth up to the boundary $\partial \Omega$ (see [15]) and can be normalized so that

$$
\sup _{\Omega} \frac{f_{2}}{f}=1, \quad \inf _{\Omega} \frac{f_{2}}{f}=-k, \text { and } 0<k \leq 1 .
$$

Let $\lambda=\lambda_{2}-\lambda_{1}$,

$$
v=\frac{f_{2} / f-(1-k) / 2}{(1+k) / 2} .
$$

Then the function $v$ satisfies the following

$$
\begin{gathered}
\Delta v=-\lambda(v+a)-2 \nabla v \nabla(\ln f) \quad \text { in } \Omega, \\
\frac{\partial v}{\partial N}=0 \quad \text { on } \partial \Omega, \\
\sup _{\Omega} v=1 \quad \text { and } \quad \inf _{\Omega} v=-1,
\end{gathered}
$$

where $N$ is the outward normal of $\partial \Omega$, and

$$
a=\frac{1-k}{1+k}
$$

is the "midrange" of the ratio $f_{2} / f$. Note that $0 \leq a<1$.

We set in this paper, unless otherwise stated,

$$
\lambda=\lambda_{2}-\lambda_{1} \quad \text { and } \quad \delta=\frac{\alpha}{\lambda} .
$$

and let

$$
Z(t)=\max _{x \in \bar{\Omega}, t=\sin ^{-1}(v(x) / b)} \frac{|\nabla v|^{2} /\left(b^{2}-v^{2}\right)}{\lambda} .
$$

for $t \in\left[-\sin ^{-1}(1 / b), \sin ^{-1}(1 / b)\right]$.

We have the following estimates (3.7) and (3.8), Lemma 3.1 and Corollaries 3.2 and 3.3.

$$
\begin{gathered}
0<\delta \leq \frac{1}{2} . \\
Z(t) \leq 1+a, \quad t \in\left[-\sin ^{-1}\left(\frac{1}{b}\right), \sin ^{-1}\left(\frac{1}{b}\right)\right] .
\end{gathered}
$$


Lemma 3.1. If the function $z:\left[-\sin ^{-1}(1 / b), \sin ^{-1}(1 / b)\right] \mapsto \mathbf{R}^{1}$ satisfies the following

(1) $z(t) \geq Z(t) \quad t \in\left[-\sin ^{-1}(1 / b), \sin ^{-1}(1 / b)\right]$,

(2) there exists some $x_{0} \in \bar{\Omega}$ such that $z\left(t_{0}\right)=Z\left(t_{0}\right)$ at point $t_{0}=\sin ^{-1}\left(v\left(x_{0}\right) / b\right)$,

(3) $z\left(t_{0}\right)>0$,

then we have the following

$$
\begin{aligned}
0 \leq & \frac{1}{2} z^{\prime \prime}\left(t_{0}\right) \cos ^{2} t_{0}-z^{\prime}\left(t_{0}\right) \cos t_{0} \sin t_{0}-z\left(t_{0}\right)+1+c \sin t_{0} \\
& -2 \delta \cos ^{2} t_{0}-\frac{z^{\prime}\left(t_{0}\right)}{4 z\left(t_{0}\right)} \cos t_{0}\left[z^{\prime}\left(t_{0}\right) \cos t_{0}-2 z\left(t_{0}\right) \sin t_{0}+2 \sin t_{0}+2 c\right] .
\end{aligned}
$$

Corollary 3.2. If in addition to the above conditions (1)-(3) in Lemma 3.1, $z^{\prime}\left(t_{0}\right) \geq 0$ and $1-c \leq z\left(t_{0}\right) \leq 1+a$, then we have the following

$$
0 \leq \frac{1}{2} z^{\prime \prime}\left(t_{0}\right) \cos ^{2} t_{0}-z^{\prime}\left(t_{0}\right) \cos t_{0} \sin t_{0}-z\left(t_{0}\right)+1+c \sin t_{0}-2 \delta \cos ^{2} t_{0}
$$

Corollary 3.3. If $a=0$, which is defined in (3.5), and if in addition to the above conditions (1)-(3) in Lemma 3.1, $z^{\prime}\left(t_{0}\right) \sin t_{0} \geq 0$ and $z\left(t_{0}\right) \leq 1$, then we have the following

$$
0 \leq \frac{1}{2} z^{\prime \prime}\left(t_{0}\right) \cos ^{2} t_{0}-z^{\prime}\left(t_{0}\right) \cos t_{0} \sin t_{0}-z\left(t_{0}\right)+1-2 \delta \cos ^{2} t_{0}
$$

The proofs of the above lemma and its corollaries are similar to those on [11]. For completeness we present the proofs in the last section. We now show the following result.

Theorem 3.4. If $a>0$ and $\mu \delta \leq\left(4 / \pi^{2}\right) a$ for a constant $\mu \in(0,1]$, then

$$
\lambda_{2}-\lambda_{1} \geq \frac{\pi^{2}}{d^{2}}+\mu \alpha
$$

Proof. Let $\mu_{\epsilon}=\mu-\epsilon>0$ for small positive constant $\epsilon$. Take $b>1$ close to 1 such that $\mu_{\epsilon} \delta<\left(4 / \pi^{2}\right) c$. Let

$$
z(t)=1+c \eta(t)+\mu_{\epsilon} \delta \xi(t)
$$


where $\xi$ and $\eta$ are the functions defined by (2.1) and (2.9), respectively. Let $\bar{I}=\left[-\sin ^{-1}(1 / b), \sin ^{-1}(1 / b)\right]$. We claim that

$$
Z(t) \leq z(t) \quad \text { for } t \in \bar{I} .
$$

By Lemmas 2.1 and 2.2, we have

$$
\begin{aligned}
& \frac{1}{2} z^{\prime \prime} \cos ^{2} t-z^{\prime} \cos t \sin t-z=-1-c \sin t+2 \mu_{\epsilon} \delta \cos ^{2} t \\
& z^{\prime}(t)>0 \\
& 0<1-\frac{a}{b}=z\left(-\frac{\pi}{2}\right) \leq z(t) \leq z\left(\frac{\pi}{2}\right)=1+\frac{a}{b} \leq 1+a,
\end{aligned}
$$

where (3.13) is due to the following.

$$
\begin{aligned}
z^{\prime}(t)=c \eta^{\prime}(t)+\mu_{\epsilon} \delta \xi^{\prime}(t) & =\mu_{\epsilon} \delta \eta^{\prime}(t)\left(\frac{c}{\mu_{\epsilon} \delta}+\frac{\xi^{\prime}(t)}{\eta^{\prime}(t)}\right) \\
& \geq \mu_{\epsilon} \delta \eta^{\prime}(t)\left(\frac{c}{\mu_{\epsilon} \delta}-\frac{\pi^{2}}{4}\right)>0
\end{aligned}
$$

Let $P \in \mathbf{R}^{1}$ and $t_{0} \in\left[-\sin ^{-1}(1 / b), \sin ^{-1}(1 / b)\right]$ such that

$$
P=\max _{t \in \bar{I}}(Z(t)-z(t))=Z\left(t_{0}\right)-z\left(t_{0}\right)
$$

Thus

$$
Z(t) \leq z(t)+P \text { for } t \in \bar{I} \text { and } Z\left(t_{0}\right)=z\left(t_{0}\right)+P .
$$

Suppose that $P>0$. Then $z+P$ satisfies the inequality in Corollary 3.2 of Lemma 3.1. Then

$$
\begin{aligned}
z\left(t_{0}\right)+P= & Z\left(t_{0}\right) \\
\leq & \frac{1}{2}(z+P)^{\prime \prime}\left(t_{0}\right) \cos ^{2} t_{0}-(z+P)^{\prime}\left(t_{0}\right) \cos t_{0} \sin t_{0}+1 \\
& +c \sin t_{0}-2 \delta \cos ^{2} t_{0} \\
= & \frac{1}{2} z^{\prime \prime}\left(t_{0}\right) \cos ^{2} t_{0}-z^{\prime}\left(t_{0}\right) \cos t_{0} \sin t_{0}+1+c \sin t_{0}-2 \delta \cos ^{2} t_{0} \\
\leq & \frac{1}{2} z^{\prime \prime}\left(t_{0}\right) \cos ^{2} t_{0}-z^{\prime}\left(t_{0}\right) \cos t_{0} \sin t_{0}+1+c \sin t_{0}-2 \mu_{\epsilon} \delta \cos ^{2} t_{0} \\
= & z\left(t_{0}\right) .
\end{aligned}
$$


This contradicts the assumption $P>0$. Thus $P \leq 0$ and (3.11) must hold. Now we have

$$
|\nabla t|^{2} \leq \lambda z(t) \quad \text { for } t \in \bar{I}
$$

that is

$$
\sqrt{\lambda} \geq \frac{|\nabla t|}{\sqrt{z(t)}}
$$

Let $q_{1}$ and $q_{2}$ be two points in $\bar{\Omega}$ such that $v\left(q_{1}\right)=-1$ and $v\left(q_{2}\right)=1$, and let $L$ be the minimum geodesic segment between $q_{1}$ and $q_{2}$. L lies on $\bar{\Omega}$ completely, since $\bar{\Omega}$ is convex. We integrate both sides of (3.16) along $L$ and change variable and let $b \rightarrow 1$. Then

$$
\begin{aligned}
\sqrt{\lambda} d & \geq \int_{L} \frac{|\nabla t|}{\sqrt{z(t)}} d l=\int_{-\pi / 2}^{\pi / 2} \frac{1}{\sqrt{z(t)}} d t \\
& \geq \frac{\left(\int_{-\pi / 2}^{\pi / 2} d t\right)^{3 / 2}}{\left(\int_{-\pi / 2}^{\pi / 2} z(t) d t\right)^{1 / 2}} \geq\left(\frac{\pi^{3}}{\int_{-\pi / 2}^{\pi / 2} z(t) d t}\right)^{1 / 2} .
\end{aligned}
$$

Square the two sides. Then

$$
\lambda \geq \frac{\pi^{3}}{d^{2} \int_{-\pi / 2}^{\pi / 2} z(t) d t}
$$

Now

$$
\int_{-\pi / 2}^{\pi / 2} z(t) d t=\int_{-\pi / 2}^{\pi / 2}\left[1+a \eta(t)+\mu_{\epsilon} \delta \xi(t)\right] d t=\left(1-\mu_{\epsilon} \delta\right) \pi
$$

where we used the facts that $\int_{-\pi / 2}^{\pi / 2} \eta(t) d t=0$ since $\eta$ is an even function, and that $\int_{-\pi / 2}^{\pi / 2} \xi(t) d t=-\pi$ (by (2.4) in Lemma 2.1). Therefore

$$
\lambda \geq \frac{\pi^{2}}{\left(1-\mu_{\epsilon} \delta\right) d^{2}} \quad \text { and } \quad \lambda \geq \frac{\pi^{2}}{d^{2}}+\mu_{\epsilon} \alpha
$$

Letting $\epsilon \rightarrow 0$, we obtain

$$
\lambda \geq \frac{\pi^{2}}{(1-\mu \delta) d^{2}} \quad \text { and } \quad \lambda \geq \frac{\pi^{2}}{d^{2}}+\mu \alpha
$$


Theorem 3.5. If the "midrange" $a=0$, then

$$
\lambda_{2}-\lambda_{1} \geq \frac{\pi^{2}}{d^{2}}+\alpha .
$$

Proof. Let

$$
y(t)=1+\delta \xi .
$$

By Lemma 2.1, for $-\pi / 2<t<\pi / 2$, we have

$$
\begin{aligned}
& \frac{1}{2} y^{\prime \prime} \cos ^{2} t-y^{\prime} \cos t \sin t-y=-1+2 \delta \cos ^{2} t, \\
& y^{\prime}(t) \sin t \geq 0,
\end{aligned}
$$

and

$$
y\left( \pm \frac{\pi}{2}\right)=1 \quad \text { and } 0<y(t)<1 .
$$

We need only show that $Z(t) \leq y(t)$ on $[-\pi / 2, \pi / 2]$. If it is not true, then there is a $t_{0}$ and a number $P>0$ such that $P=Z\left(t_{0}\right)-y\left(t_{0}\right)=\max Z(t)-$ $y(t)$. Note that $y(t)+P \geq 1-(1 / 2)\left(\pi^{2} / 4-1\right)+P>0$. So $y+P$ satisfies the inequality in Corollary 3.3 in Lemma 3.1. Therefore

$$
\begin{aligned}
y\left(t_{0}\right)+P & =Z\left(t_{0}\right) \\
& \leq \frac{1}{2}(y+P)^{\prime \prime}\left(t_{0}\right) \cos ^{2} t_{0}-(y+P)^{\prime}\left(t_{0}\right) \cos t_{0} \sin t_{0}+1-2 \delta \cos ^{2} t_{0} \\
& =\frac{1}{2} y^{\prime \prime}\left(t_{0}\right) \cos ^{2} t_{0}-y^{\prime}\left(t_{0}\right) \cos t_{0} \sin t_{0}+1-2 \delta \cos ^{2} t_{0} \\
& =y\left(t_{0}\right) .
\end{aligned}
$$

This contradicts the assumption $P>0$. The rest of the proof is similar to that of Theorem 3.4, just noticing that $\delta \leq 1 / 2<4 /\left(\pi^{2}-4\right)$.

Proof of Theorem 1.2. Since $a=0$ in such case, we may directly apply Theorem 3.5.

Proof of Theorem 1.1. Since $0 \leq a<1$, either $a=0$ or $0<a<1$.

If $a=0$, then we apply Theorem 3.5 to obtain the bound with $\mu=1$,

$$
\lambda \geq \frac{\pi^{2}}{d^{2}}+\alpha .
$$

If $0<a<1$, then there are several cases altogether. 
- (I): $a \geq\left(\pi^{2} / 4\right) \delta$.

- (II): $a<\left(\pi^{2} / 4\right) \delta$.

- (II-a): $a \geq 0.765$.

- (II-b): $0<a<0.765$.

* (II-b-1): $a \geq 1.53 \delta$.

* (II-b-2): $a<1.53 \delta$.

For Case (I): $\quad 0<a<1$ and $a \geq\left(\pi^{2} / 4\right) \delta$, we apply Theorem 3.5 for $\mu=1$ to get the following lower bound

$$
\frac{\pi^{2}}{d^{2}}+\alpha
$$

For Case (II-a): $0.765 \leq a<\left(\pi^{2} / 4\right) \delta$, we apply Theorem 3.4 with $\mu=$ $\left(4 / \pi^{2}\right)(a / \delta)$ since $\left(\left(4 / \pi^{2}\right)(a / \delta)\right) \delta \leq\left(4 / \pi^{2}\right) a$ and $0<\left(4 / \pi^{2}\right)(a / \delta)<1$. Then

$$
\lambda \geq \frac{\pi^{2}}{d^{2}}+\frac{4}{\pi^{2}} \frac{a}{\delta} \alpha=\frac{\pi^{2}}{d^{2}}+\frac{4 a}{\pi^{2}} \lambda .
$$

Thus

$$
\lambda \geq \frac{1}{1-\left(4 a / \pi^{2}\right)} \frac{\pi^{2}}{d^{2}} .
$$

On the other hand we have bound (3.7),

$$
\lambda \geq 2 \alpha .
$$

The above two estimates give

$$
\lambda \geq \frac{\pi^{2}}{d^{2}}+\frac{4 a}{\pi^{2}} 2 \alpha \geq \frac{\pi^{2}}{d^{2}}+\frac{8(0.765)}{\pi^{2}} \alpha>\frac{\pi^{2}}{d^{2}}+\frac{31}{50} \alpha .
$$

The theorem is proved in this case.

For Case (II-b-1): $0<a<0.765, a<\left(\pi^{2} / 4\right) \delta$ and $a \geq 1.53 \delta$, we apply Theorem 3.4 with $\mu=\left(4 / \pi^{2}\right)(a / \delta)$ since $\left(\left(4 / \pi^{2}\right)(a / \delta)\right) \delta \leq\left(4 / \pi^{2}\right) a$ and $0<$ $\left(4 / \pi^{2}\right)(a / \delta)<1$. Then

$$
\lambda \geq \frac{\pi^{2}}{d^{2}}+\frac{4}{\pi^{2}} \frac{a}{\delta} \alpha \geq \frac{\pi^{2}}{d^{2}}+\frac{4}{\pi^{2}} \frac{153}{100} \alpha>\frac{\pi^{2}}{d^{2}}+\frac{31}{50} \alpha,
$$

which is what we want to prove. 
For the remaining Case (II-b-2): $0<a<0.765, \quad a<\left(\pi^{2} / 4\right) \delta$ and $a<1.53 \delta$, we define a function $z$ by

$$
z(t)=1+c \eta(t)+\left(\delta-\sigma c^{2}\right) \xi(t) \quad \text { on }\left[-\sin ^{-1} \frac{1}{b}, \sin ^{-1}\right]
$$

where

$$
\begin{gathered}
\sigma=\frac{\tau}{\left(\left[3 / 2-\pi^{2} / 8-\left(\pi^{2} / 32-1 / 6\right)(153 / 100)\right] 200 / 153\right.} \\
\left.-\frac{(8 / 3 \pi-\pi / 4)^{2}}{\left[-1+\left(12-\pi^{2}\right)(100 / 153)\right]}\right) c
\end{gathered}
$$

and

$$
\tau=\frac{2}{3 \pi^{2}}\left(\frac{4}{3(4-\pi)}+\frac{3(4-\pi)}{4}-2\right)
$$

Let $\bar{I}=\left[-\sin ^{-1}(1 / b), \sin ^{-1}(1 / b)\right]$. We now show that

$$
Z(t) \leq z(t) \text { on } \bar{I}
$$

If (3.24) is not true, then there exist a constant $P>0$ and $t_{0}$ such that

$$
P c^{2}=\frac{Z\left(t_{0}\right)-z\left(t_{0}\right)}{-\xi\left(t_{0}\right)}=\max _{t \in\left[-\sin ^{-1}(1 / b), \sin ^{-1}(1 / b)\right]} \frac{Z(t)-z(t)}{-\xi(t)} .
$$

Let $w(t)=z(t)-P c^{2} \xi(t)=1+c \eta(t)+m \xi(t)$, where $m=\delta-\sigma c^{2}-P c^{2}$. Then

$$
Z(t) \leq w(t) \text { on } \bar{I} \quad \text { and } \quad Z\left(t_{0}\right)=w\left(t_{0}\right)
$$

By Lemma 4.1, $w\left(t_{0}\right)>0$. So $w$ satisfies (3.9) in Lemma 3.1,

$$
0 \leq-2(\sigma+P) c^{2} \cos ^{2} t_{0}-\frac{w^{\prime}\left(t_{0}\right)}{4 w\left(t_{0}\right)} \cos t_{0}\left(\frac{8 c}{\pi} \cos t+4 m t \cos t\right)
$$

We used (2.2), (2.3), (2.10) and (2.11) to obtain the above inequality. Thus $(3.25)$

$$
\sigma+P \leq-\frac{w^{\prime}\left(t_{0}\right)}{2 c^{2} w\left(t_{0}\right)}\left(\frac{2 c}{\pi}+m t\right)=-\frac{\eta^{\prime}\left(t_{0}\right)}{\pi w\left(t_{0}\right)}\left(1+\frac{m \xi^{\prime}\left(t_{0}\right)}{c \eta^{\prime}\left(t_{0}\right)}\right)\left(1+\frac{\pi m}{2 c} t_{0}\right) .
$$


The right-hand side is not positive for $t_{0} \geq 0$, by Lemmas 2.1 and 2.2. Thus $t_{0}<0$, and

$$
\begin{aligned}
-\left(1+\frac{m \xi^{\prime}\left(t_{0}\right)}{c \eta^{\prime}\left(t_{0}\right)}\right)\left(1+\frac{\pi m}{2 c} t_{0}\right)= & \frac{2 \xi^{\prime}\left(t_{0}\right)}{\pi t_{0} \eta^{\prime}\left(t_{0}\right)}\left(\frac{\pi t_{0} \eta^{\prime}\left(t_{0}\right)}{2 \xi^{\prime}\left(t_{0}\right)}+\frac{\pi m}{2 c} t_{0}\right) \\
& \times\left(-1-\frac{\pi m}{2 c} t_{0}\right) \\
\leq & \frac{1}{4} \frac{2 \xi^{\prime}\left(t_{0}\right)}{\pi t_{0} \eta^{\prime}\left(t_{0}\right)}\left(\frac{\pi t_{0} \eta^{\prime}\left(t_{0}\right)}{2 \xi^{\prime}\left(t_{0}\right)}-1\right)^{2} \\
& =\frac{1}{4}\left(\frac{2 \xi^{\prime}\left(t_{0}\right)}{\pi t_{0} \eta^{\prime}\left(t_{0}\right)}+\left(\frac{2 \xi^{\prime}\left(t_{0}\right)}{\pi t_{0} \eta^{\prime}\left(t_{0}\right)}\right)^{-1}-2\right) .
\end{aligned}
$$

By Lemmas 2.1 and 2.2, we have $2\left(3-\pi^{2} / 4\right) \leq \xi^{\prime}(t) / t \leq 4 / 3$ and $2(4 / \pi-1) \leq \eta^{\prime}(t) \leq 8 / 3 \pi$. So

$$
\frac{3\left(12-\pi^{2}\right)}{8} \leq \frac{2 \xi^{\prime}\left(t_{0}\right)}{\pi t_{0} \eta^{\prime}\left(t_{0}\right)} \leq \frac{4}{3(4-\pi)}
$$

Note that the function $f(t)=t+1 / t-2$ achieves it maximum on $[A, B]$ not containing 0 at an endpoint. Therefore

$$
\left|-\left(1+\frac{m \xi^{\prime}\left(t_{0}\right)}{c \eta^{\prime}\left(t_{0}\right)}\right)\left(1+\frac{\pi m}{2 c} t_{0}\right)\right| \leq \frac{1}{4}\left(\frac{4}{3(4-\pi)}+\frac{3(4-\pi)}{4}-2\right) .
$$

Now (3.25) becomes

$$
\sigma+P \leq \frac{\tau}{w\left(t_{0}\right)}
$$

where $\tau$ is the number in (3.23). On the other hand, by Lemma 4.1,

$$
\begin{aligned}
z\left(t_{0}\right) & \geq\left(\left[\frac{3}{2}-\frac{\pi^{2}}{8}-\left(\frac{\pi^{2}}{32}-\frac{1}{6}\right) \frac{153}{100}\right] \frac{200}{153}-\frac{(8 / 3 \pi-\pi / 4)^{2}}{\left[-1+\left(12-\pi^{2}\right)(100 / 153)\right]}\right) c \\
& =\frac{\tau}{\sigma}>0 .
\end{aligned}
$$

Since $-P \xi\left(t_{0}\right) \geq 0$, we have $w\left(t_{0}\right) \geq z\left(t_{0}\right)$. This fact, (3.26) and (3.27) imply that for $P>0$

$$
\sigma+P<\sigma
$$

which is impossible. 
Therefore we have the estimate (3.24). Now we proceed as in the proof of Theorem 3.4. We obtain the following

$$
\lambda d^{2} \geq \frac{\pi^{3}}{\pi\left[1-\left(\delta-\sigma c^{2}\right)\right]} .
$$

Since $\delta-\sigma c^{2}>0.625 \delta$ by Lemma 4.1 , we have

$$
\lambda \geq \frac{1}{\left[1-\left(\delta-\sigma c^{2}\right)\right]} \frac{\pi^{2}}{d^{2}} \geq \frac{1}{[1-0.625 \delta]} \frac{\pi^{2}}{d^{2}}
$$

and

$$
\lambda \geq \frac{\pi^{2}}{d^{2}}+0.625 \alpha>\frac{\pi^{2}}{d^{2}}+\frac{31}{50} \alpha .
$$

\section{Technical lemmas and estimates}

Lemma 4.1. If $a<1.53 \delta$ and $0<a<0.765$ then

$$
\begin{aligned}
z(t) & =1+c \eta(t)+\delta \xi(t) \\
& \geq\left(\left[\frac{3}{2}-\frac{\pi^{2}}{8}-\left(\frac{\pi^{2}}{32}-\frac{1}{6}\right) \frac{153}{100}\right] \frac{200}{153}-\frac{(8 / 3 \pi-\pi / 4)^{2}}{\left[-1+\left(12-\pi^{2}\right)(100 / 153)\right]}\right) c \\
& >0,
\end{aligned}
$$

for $t \in[-\pi / 2, \pi / 2]$ and

$$
\delta-\sigma c^{2} \approx 0.625162283437 \delta>0.625 \delta
$$

where $c=a / b$ and $b>1$ is any constant and $\sigma$ is the constant in (3.22).

Proof. By Lemmas 1.3-1.5, the function $z$ on $[-\pi / 2, \pi / 2]$ has a unique critical point $t_{1} \in(-\pi / 2,0)$ if $0<a<\left(\pi^{2} / 4\right) \delta$ and $z$ is decreasing on $\left[-\pi / 2, t_{1}\right]$ and increasing on $\left[t_{1}, \pi / 2\right]$. Therefore

$$
\min _{[-\pi / 2, \pi / 2]} z=\min _{[-\pi / 2,0]} z=z\left(t_{1}\right)
$$

So we need only consider the restricted function $\left.z\right|_{[-\pi / 2,0]}$ for the minimum.

Now first consider the Taylor expansion of $\xi$ at 0 for $t \in[-\pi / 2,0]$. By Lemma 2.1, $\xi(0)=-\pi^{2} / 4+1, \xi^{\prime}(0)=0$ and $\xi^{\prime \prime}(0)=2\left(3-\pi^{2} / 4\right)$ and 
$\xi^{\prime \prime \prime}(t)<0$ on $(-\pi / 2,0)$. Thus

$$
\xi(t) \geq-\left(\frac{\pi^{2}}{4}-1\right)+\left(3-\frac{\pi^{2}}{4}\right) t^{2} .
$$

Similarly, using the data $\eta(-\pi / 2)=-1, \eta^{\prime}(-\pi / 2)=8 / 3 \pi$ and $\eta^{\prime \prime \prime}(t)>0$ on $(-\pi / 2,0)$ (actually on $[-\pi / 2, \pi / 2])$, and the Taylor expansion of $\eta$ at $-\pi / 2$, we have for $t \in[-\pi / 2,0]$,

$$
\eta(t) \geq-\left(\frac{\pi^{2}}{16}-\frac{1}{3}\right)+\left(\frac{8}{3 \pi}-\frac{\pi}{4}\right) t-\frac{1}{4} t^{2} .
$$

Therefore on $[-\pi / 2,0]$,

$$
\begin{aligned}
z(t)= & 1+c \eta(t)+\delta \xi(t) \\
\geq & 1-\left(\frac{\pi^{2}}{16}-\frac{1}{3}\right) c-\left(\frac{\pi^{2}}{4}-1\right) \delta+\left(\frac{8}{3 \pi}-\frac{\pi}{4}\right) c t \\
& +\left[-\frac{1}{4} c+\left(3-\frac{\pi^{2}}{4}\right) \delta\right] t^{2}
\end{aligned}
$$

Let $\nu=1.53$ and $a_{0}=0.765$. That $a \leq \nu \delta$ implies $c=a / b<\nu \delta$, where $b>1$ is a constant. Using conditions $(3.7) \delta \leq(n-1) / 2 n<1 / 2$ and $a \leq a_{0}$, we obtain

$$
\begin{aligned}
1-\left(\frac{\pi^{2}}{16}-\frac{1}{3}\right) c-\left(\frac{\pi^{2}}{4}-1\right) \delta & \geq 1-\left(\frac{\pi^{2}}{16}-\frac{1}{3}\right) \nu \delta-\left(\frac{\pi^{2}}{4}-1\right) \delta \\
& \geq \frac{3}{2}-\frac{\pi^{2}}{8}-\left(\frac{\pi^{2}}{32}-\frac{1}{6}\right) \nu \\
& >\left(\frac{3}{2}-\frac{\pi^{2}}{8}-\left(\frac{\pi^{2}}{32}-\frac{1}{6}\right) \nu\right) \frac{1}{a_{0}} c
\end{aligned}
$$

and

$$
\begin{aligned}
1+ & \multicolumn{1}{l}{(t)+\delta \xi(t)} \\
\geq & \left(\frac{3}{2}-\frac{\pi^{2}}{8}-\left(\frac{\pi^{2}}{32}-\frac{1}{6}\right) \nu\right) \frac{1}{a_{0}} c+\left(\frac{8}{3 \pi}-\frac{\pi}{4}\right) c t \\
& +\left[-\frac{1}{4} c+\left(3-\frac{\pi^{2}}{4}\right) \frac{1}{\nu} c\right] t^{2}
\end{aligned}
$$




$$
\begin{aligned}
= & \left(\left[\frac{3}{2}-\frac{\pi^{2}}{8}-\left(\frac{\pi^{2}}{32}-\frac{1}{6}\right) \nu\right] \frac{1}{a_{0}}+\left(\frac{8}{3 \pi}-\frac{\pi}{4}\right) t\right. \\
& \left.+\left[-\frac{1}{4}+\left(3-\frac{\pi^{2}}{4}\right) \frac{1}{\nu}\right] t^{2}\right) c \\
\geq & \left(\left[\frac{3}{2}-\frac{\pi^{2}}{8}-\left(\frac{\pi^{2}}{32}-\frac{1}{6}\right) \nu\right] \frac{1}{a_{0}}-\frac{(8 / 3 \pi-\pi / 4)^{2}}{4\left[-1 / 4+\left(3-\pi^{2} / 4\right)(1 / \nu)\right]}\right) c \\
\geq & \left(\left[\frac{3}{2}-\frac{\pi^{2}}{8}-\left(\frac{\pi^{2}}{32}-\frac{1}{6}\right) \nu\right] \frac{1}{a_{0}}-\frac{(8 / 3 \pi-\pi / 4)^{2}}{\left[-1+\left(12-\pi^{2}\right)(1 / \nu)\right]}\right) c \\
> & 0.5433>0 .
\end{aligned}
$$

Let $\tau$ be the constant in (3.23). Then

$$
\begin{aligned}
\sigma c^{2} & =\frac{\tau c}{\left(\left[3 / 2-\pi^{2} / 8-\left(\pi^{2} / 32-1 / 6\right) \nu\right]\left(1 / a_{0}\right)-\frac{(8 / 3 \pi-\pi / 4)^{2}}{\left[-1+\left(12-\pi^{2}\right)(1 / \nu)\right]}\right)}, \\
& \leq \frac{\tau \nu \delta}{\left(\left[3 / 2-\pi^{2} / 8-\left(\pi^{2} / 32-1 / 6\right) \nu\right]\left(1 / a_{0}\right)-\frac{(8 / 3 \pi-\pi / 4)^{2}}{\left[-1+\left(12-\pi^{2}\right)(1 / \nu)\right]}\right)} \\
& \approx 0.374837516563 \delta
\end{aligned}
$$

and

$$
\delta-\sigma c^{2}>0.625 \delta .
$$

Proof of the estimate (3.7). We estimate the maximum of the function

$$
P(x)=|\nabla v|^{2}+A v^{2},
$$

where $v$ is the function in (3.1), and where $A \geq 0$ is a constant.

Let $A=0$ in (4.1). Function $P$ must achieve its maximum at some point $x_{0} \in \bar{\Omega}$. Suppose that $x_{0} \in \partial \Omega$. Choose an orthornormal frame $\left\{e_{1}, \ldots, e_{n}\right\}$ about $x_{0}$ such that $e_{n}$ is a outward normal to $\partial \Omega$. By (3.3), $v_{n}=\partial v / \partial N=0$. Thus at $x_{0}$

$$
\begin{aligned}
v_{i n} & =e_{i} e_{n} v-\left(\nabla_{e_{i}} e_{n}\right) v \\
& =-\left(\nabla_{e_{i}} e_{n}\right) v \\
& =-\sum_{j=1}^{n-1} h_{i j} v_{j}
\end{aligned}
$$


and

$$
\begin{aligned}
P_{n} & =2 \sum_{j=1}^{n} v_{j} v_{j n}+2 A v v_{n}=\sum_{j=1}^{n-1} v_{j} v_{j n} \\
& =-2 \sum_{i, j=1}^{n-1} h_{i j} v_{i} v_{j}
\end{aligned}
$$

$\leq 0$ by the convexity of $\partial \Omega$.

On the other hand, that $P$ attains the maximum at the boundary point $x_{0}$ implies that

$$
P_{n} \geq 0 \text {. }
$$

Thus at $x_{0},-2 \sum_{i, j=1}^{n-1} h_{i j} v_{i} v_{j}=P_{n}=0$. By the convexity, $v_{1}=\cdots=v_{n-1}=$ 0 and $\nabla v=0$ at $x_{0}$. Therefore $v$ is a constant. This is impossible, so $x_{0} \in \Omega$. $\nabla v\left(x_{0}\right) \neq 0$ (otherwise $v$ is a constant). At $x_{0}$,

$$
\nabla P\left(x_{0}\right)=0 \quad \text { and } \quad \Delta P\left(x_{0}\right) \leq 0 .
$$

Take a local frame so that

$$
v_{1}\left(x_{0}\right)=\nabla v\left(x_{0}\right) \quad \text { and } \quad v_{i}\left(x_{0}\right)=0, \quad i \geq 2 .
$$

Thus at $t_{0}$ we have

$$
\begin{gathered}
0=\frac{1}{2} \nabla P_{i}=v_{j} v_{j i}+A v v_{i} \\
v_{11}=-A v \quad \text { and } \quad v_{1 i}=0 \quad i \geq 2,
\end{gathered}
$$

and

$$
\begin{aligned}
0 \geq & \frac{1}{2} \Delta P\left(x_{0}\right) \\
= & v_{j i} v_{j i}+v_{j} v_{j i i}+A v_{i} v_{i}+A v v_{i i} \\
= & v_{j i}^{2}+v_{1}(\Delta v)_{1}+R_{j i} v_{j} v_{i}+A|\nabla v|^{2}+A v \Delta v \\
\geq & v_{11}^{2}+v_{1}(\Delta v)_{1}+A|\nabla v|^{2}+A v \Delta v \\
= & (-A v)^{2}-\lambda|\nabla v|^{2}-2 v_{1}(\nabla v \nabla \ln f)_{1}+A|\nabla v|^{2}-\lambda A v(v+a) \\
& -2 A v \nabla v \nabla \ln f \\
= & -(\lambda-A)|\nabla v|^{2}-A v^{2}(\lambda-A)-a \lambda A v-2 v_{1}^{2}(\ln f)_{11} \\
& -2 v_{1}(\ln f)_{1}\left(v_{11}+A v\right),
\end{aligned}
$$


where we have used (3.6) and (4.2). Therefore at $x_{0}$,

$$
0 \geq-(\lambda-2 \alpha-A)|\nabla v|^{2}-A(\lambda-A) v^{2}-a \lambda A v
$$

Using the fact that $A=0$ in the above inequality, we get (3.7).

Proof of the estimate (3.8). We first prove the following

$$
\frac{|\nabla v|^{2}}{b^{2}-v^{2}} \leq \lambda(1+a)
$$

where $b>1$ is an arbitrary constant.

Let $A=\lambda(1+a)+\epsilon$ in (4.1) for small $\epsilon>0$. The function $P$ achieves its maximum at some $x_{0} \in \bar{\Omega}$. If $\nabla v\left(x_{0}\right) \neq 0$ and $x_{0} \in \Omega$, then (4.3) holds at $x_{0}$ with $A=\lambda(1+a)+\epsilon$. Thus

$$
\left|\nabla v\left(x_{0}\right)\right|^{2}+\lambda(1+a) v\left(x_{0}\right)^{2} \leq \frac{a \lambda v}{a \lambda+\epsilon}[\lambda(1+a)+\epsilon] \leq[\lambda(1+a)+\epsilon] .
$$

This estimate holds if $x_{0} \in \bar{\Omega}$ with $\nabla v\left(x_{0}\right)=0$. If $x_{0} \in \partial \Omega$, then the convexity of $\Omega$ and previous argument in the proof of (3.7) imply that the above estimate holds. So we have the estimate (4.4). By the definition of $Z$, we have (3.8).

Proof of Lemma 3.1. Define

$$
J(x)=\left\{\frac{|\nabla v|^{2}}{b^{2}-v^{2}}-\lambda z\right\} \cos ^{2} t,
$$

where $t=\sin ^{-1}(v(x) / b)$. Then

$$
J(x) \leq 0 \text { for } x \in \bar{\Omega} \text { and } \quad J\left(x_{0}\right)=0 .
$$

If $\nabla v\left(x_{0}\right)=0$ then

$$
0=J\left(x_{0}\right)=-\lambda z \cos ^{2} t .
$$

This contradicts condition (1.3) in the theorem. Therefore

$$
\nabla v\left(x_{0}\right) \neq 0
$$

If $x_{0} \in \partial \Omega$, then by an argument in the proof of (3.7), the convexity of $\Omega$ and that $J\left(x_{0}\right)$ is the maximum would imply that $\nabla v\left(x_{0}\right)=0$. Thus 
$x_{0} \in \Omega=\bar{\Omega} \backslash \partial \Omega$. The Maximum Principle implies that

$$
\nabla J\left(x_{0}\right)=0 \quad \text { and } \quad \Delta J\left(x_{0}\right) \leq 0
$$

$J(x)$ can be rewritten as

$$
J(x)=\frac{1}{b^{2}}|\nabla v|^{2}-\lambda z \cos ^{2} t
$$

Thus (4.5) is equivalent to

$$
\left.\frac{2}{b^{2}} \sum_{i} v_{i} v_{i j}\right|_{x_{0}}=\left.\lambda \cos t\left[z^{\prime} \cos t-2 z \sin t\right] t_{j}\right|_{x_{0}}
$$

and

$$
\begin{aligned}
0 \geq & \frac{2}{b^{2}} \sum_{i, j} v_{i j}^{2}+\frac{2}{b^{2}} \sum_{i, j} v_{i} v_{i j j}-\lambda\left(z^{\prime \prime}|\nabla t|^{2}+z^{\prime} \Delta t\right) \cos ^{2} t \\
& +4 \lambda z^{\prime} \cos t \sin t|\nabla t|^{2}-\left.\lambda z \Delta \cos ^{2} t\right|_{x_{0}}
\end{aligned}
$$

Choose a normal coordinate around $x_{0}$ such that $v_{1}\left(x_{0}\right) \neq 0$ and $v_{i}\left(x_{0}\right)=0$ for $i \geq 2$. Then (4.6) implies

$$
\left.v_{11}\right|_{x_{0}}=\left.\frac{\lambda b}{2}\left(z^{\prime} \cos t-2 z \sin t\right)\right|_{x_{0}} \text { and }\left.\quad v_{1 i}\right|_{x_{0}}=0 \text { for } i \geq 2
$$

Now we have

$$
\begin{aligned}
\left.|\nabla v|^{2}\right|_{x_{0}} & =\left.\lambda b^{2} z \cos ^{2} t\right|_{x_{0}}, \\
\left.|\nabla t|^{2}\right|_{x_{0}} & =\frac{|\nabla v|^{2}}{b^{2}-v^{2}}=\left.\lambda z\right|_{x_{0}}, \\
\left.\frac{\Delta v}{b}\right|_{x_{0}} & =\Delta \sin t=\cos t \Delta t-\left.\sin t|\nabla t|^{2}\right|_{x_{0}}, \\
\left.\Delta t\right|_{x_{0}} & =\frac{1}{\cos t}\left(\sin t|\nabla t|^{2}+\frac{\Delta v}{b}\right) \\
& =\left.\frac{1}{\cos t}\left[\lambda z \sin t-\frac{\lambda}{b}(v+a)-\frac{2}{b} v_{1}(\ln f)_{1}\right]\right|_{x_{0}},
\end{aligned}
$$


and

$$
\begin{aligned}
\left.\Delta \cos ^{2} t\right|_{x_{0}} & =\Delta\left(1-\frac{v^{2}}{b^{2}}\right)=-\frac{2}{b^{2}}|\nabla v|^{2}-\frac{2}{b^{2}} v \Delta v \\
& =-2 \lambda z \cos ^{2} t+\frac{2}{b^{2}} \lambda v(v+a)+\left.\frac{4}{b^{2}} v v_{1}(\ln f)_{1}\right|_{x_{0}}
\end{aligned}
$$

Therefore,

$$
\begin{aligned}
\left.\frac{2}{b^{2}} \sum_{i, j} v_{i j}^{2}\right|_{x_{0}} \geq & \frac{2}{b^{2}} v_{11}^{2} \\
= & \frac{\lambda^{2}}{2}\left(z^{\prime}\right)^{2} \cos ^{2} t-2 \lambda^{2} z z^{\prime} \cos t \sin t+\left.2 \lambda^{2} z^{2} \sin ^{2} t\right|_{x_{0}}, \\
\left.\frac{2}{b^{2}} \sum_{i, j} v_{i} v_{i j j}\right|_{x_{0}}= & \frac{2}{b^{2}}(\nabla v \nabla(\Delta v)+R(\nabla v, \nabla v)) \\
\geq & \frac{2}{b^{2}} \nabla v \nabla(\Delta v) \\
= & -2 \lambda^{2} z \cos ^{2} t-\frac{4}{b^{2}} v_{1} v_{11}(\ln f)_{1}-\left.\frac{4}{b^{2}} v_{1}^{2}(\ln f)_{11}\right|_{x_{0}}, \\
& -\left.\lambda\left(z^{\prime \prime}|\nabla t|^{2}+z^{\prime} \Delta t\right) \cos { }^{2} t\right|_{x_{0}} \\
= & -\lambda^{2} z z^{\prime \prime} \cos ^{2} t-\lambda^{2} z z^{\prime} \cos t \sin t+\frac{1}{b} \lambda^{2} z^{\prime}(v+a) \cos t \\
& +\left.\frac{2}{b} \lambda z^{\prime} v_{1}(\ln f)_{1} \cos t\right|_{x_{0}},
\end{aligned}
$$

and

$$
\begin{aligned}
4 \lambda z^{\prime} \cos t \sin t|\nabla t|^{2}-\left.\lambda z \Delta \cos ^{2} t\right|_{x_{0}} \\
=4 \lambda^{2} z z^{\prime} \cos t \sin t+2 \lambda^{2} z^{2} \cos ^{2} t-\frac{2}{b} \lambda^{2} z \sin t(v+a) \\
\quad-\left.\frac{4}{b} \lambda z \sin t v_{1}(\ln f)_{1}\right|_{x_{0}} .
\end{aligned}
$$

Putting these results into (4.7) we obtain

$$
\begin{aligned}
0 \geq & -\lambda^{2} z z^{\prime \prime} \cos ^{2} t+\frac{\lambda^{2}}{2}\left(z^{\prime}\right)^{2} \cos ^{2} t+\lambda^{2} z^{\prime} \cos t(z \sin t+c+\sin t) \\
& +2 \lambda^{2} z^{2}-2 \lambda^{2} z-2 \lambda^{2} c z \sin t-4 \lambda z \cos ^{2} t(\ln f)_{11} \\
& -\left.\frac{4}{b^{2}}\left[v_{11}-\frac{\lambda b}{2}\left(z^{\prime} \cos t-2 z \sin t\right)\right] v_{1}(\ln f)_{1}\right|_{x_{0}}
\end{aligned}
$$


The last term in (4.9) is 0 due to (4.8). Now

$$
z\left(t_{0}\right)>0
$$

by condition (1.3) in the theorem, and

$$
-\frac{(\ln f)_{11}}{\lambda} \geq \delta
$$

by the definition of $\delta$. Dividing two sides of (4.9) by $\left.2 \lambda^{2} z\right|_{x_{0}}$ and taking (4.11) into account, we have

$$
\begin{aligned}
0 \geq & -\frac{1}{2} z^{\prime \prime}\left(t_{0}\right) \cos ^{2} t_{0}+\frac{1}{2} z^{\prime}\left(t_{0}\right) \cos t_{0}\left(\sin t_{0}+\frac{c+\sin t_{0}}{z\left(t_{0}\right)}\right)+z\left(t_{0}\right) \\
& -1-c \sin t_{0}+2 \delta \cos ^{2} t_{0}+\frac{1}{4 z\left(t_{0}\right)}\left(z^{\prime}\left(t_{0}\right)\right)^{2} \cos ^{2} t_{0}
\end{aligned}
$$

Therefore,

$$
\begin{aligned}
0 \geq & -\frac{1}{2} z^{\prime \prime}\left(t_{0}\right) \cos ^{2} t_{0}+z^{\prime}\left(t_{0}\right) \cos t_{0} \sin t_{0}+z\left(t_{0}\right)-1-c \sin t_{0}+2 \delta \cos ^{2} t_{0} \\
& +\frac{z^{\prime}\left(t_{0}\right)}{4 z\left(t_{0}\right)} \cos t_{0}\left[z^{\prime}\left(t_{0}\right) \cos t_{0}-2 z\left(t_{0}\right) \sin t_{0}+2 \sin t_{0}+2 c\right] .
\end{aligned}
$$

Proof of Corollary 3.2. By condition (1.2) in the theorem, (3.8), $\left|\sin t_{0}\right|=$ $\left|v\left(t_{0}\right) / b\right| \leq 1 / b$ and $1-c \leq z\left(t_{0}\right) \leq 1+a$. Thus for $t_{0} \geq 0$,

$$
\begin{aligned}
-z\left(t_{0}\right) \sin t_{0}+\sin t_{0}+c & \geq-\sin t_{0}-a \sin t_{0}+\sin t_{0}+c \\
& \geq a\left(\frac{1}{b}-\sin t_{0}\right) \geq 0,
\end{aligned}
$$

and for $t_{0}<0$,

$$
-z\left(t_{0}\right) \sin t_{0}+\sin t_{0}+c \geq-\sin t_{0}+c \sin t_{0}+\sin t_{0}+c \geq c\left(1+\sin t_{0}\right) \geq 0 .
$$

In any case the last term in (3.9) is non-negative.

Proof of Corollary 3.3. The last term in (3.9) is non-negative. 


\section{References}

[1] M. Ashbaugh, Some eigenvalue comparison results for domains in $S^{n}$ and for annular domains in $R^{n+1}$, The Erwin Schödinger International Institute for Mathematical Physics — Workshop on Geometrical Aspects of Spectral Theory, eds. Leonid Friedlander and Thomas Hoffmann-Ostenhof, ftp://ftp.esi.ac.at/pub/Preprints/esi768.pdf, East Tyola, 1999.

[2] M. Ashbaugh and R. Benguria, Optimal lower bounds for the gap between the first two eigenvalues of one dimensional shrödinger operators with symmetric single-well potentials, Proc. Amer. Math. Soc. 105 (1989), 419-424.

[3] R. Bañuelos and P. Kröger, Gradient estimates for ground state Schrodinger operators and applications, Comm. Math. Phys. 224 (2002), 545-550.

[4] R. Bañuelos and P.J. Méndez-Hernández, Sharp inequalities for rations of heat kernels of Schrödinger operators and applications to spectral gaps, J. Funct. Anal. 176 (2000), 368-399.

[5] M. van den Berg, On condensation in the free-boson gas and the spectrum of Laplacian, J. Statist. Phys. 31 (1983), 623-637.

[6] H. Brascamp and E. Lieb, On extensions of the Brunn-Minkowski and Prékopa-Leindler theorems, including inequalities for log concave functions, and with an application to the diffusion equation, J. Funct. Anal. 22 (1976), 366-389.

[7] I. Chavel, Eigenvalues in Riemannian Geometry, Academic Press, Orlando, Fla, 1984.

[8] B. Davis, On the spectral gap for fixed membranes, Ark. Mat. 39 (2001), 65-74.

[9] Y.I. Lee and A.N. Wang, Estimate of $\lambda_{2}-\lambda_{1}$ on spheres, Chinese J. Math. 15 (1987), 95-97.

[10] P. Li and S.T. Yau, On the Schrödinger equation and the eigenvalue problem, Comm. Math. Phys. 88 (1983), 309-318.

[11] Jun Ling, A lower bound for the gap between the first two eigenvalues of Schrödinger operators on convex domains in $S^{n}$ or $R^{n}$, Michigan Math. J. 40 (1983), 259-270. 
[12] — A Bound for the First Fundamental Gap, PhD dissertation, State University of New York at Buffalo, 1998.

[13] R. Schoen and S.T. Yau, Lecture notes on differential geometry, Conference Proceedings and Lecture Notes in Geometry and Topology, vol. 1, International Press, 1994.

[14] R.G. Smits, Spectral gaps and rates to equilibrium for diffusions in convex domains, Michigan Math. J. 43(1) (1996), 141-157.

[15] I.M. Singer, B. Wong, S.T. Yau, and S.S.T. Yau, An estimate of the gap of the first two eigenvalues, Ann. Scuola Norm. Sup. Pisa, CI. Sci. 12 (1985), 319-333.

[16] S.T. Yau, ed., Problem section, seminar on differential geometry, Princeton University Press, Princeton, 1982.

[17] S.T. Yau, An estimate of the gap of the first two eigenvalues in the Schrödinger operator, in Lectures on Partial Differential Equations: Proceedings in honor of Louis Nirenberg's 75th Birthday, SunYung Alice Chang, eds. Chang-Shou Lin and Horng-Tzer Yau, International Press, 2003, 223-235.

[18] - Perspectives on Geometric Analysis, Preprint, 2006, arXiv: math.DG/0602363.

[19] Q.H. Yu and J.-Q. Zhong, Lower bounds of the gap between the first and second eigenvalues in the Schrödinger operators, Trans. Amer. Math. Sco. 294 (1986), 341-349.

Department of Mathematics

UTAH VALLEY UNIVERSITY

800 W UNIVERSITY PARKWAY

OREM, UT 84058

USA

E-mail address: lingju@uvu.edu

Received April 5, 2006 
